\title{
British-English norms and naming times for a set of 539 pictures: The role of age of acquisition
}

\author{
ROBERT A. JoHnSton \\ University of Kent, Canterbury, England \\ and University of Birmingham, Birmingham, England \\ KeVin Dent and Glyn W. Humphreys \\ University of Birmingham, Birmingham, England \\ AND \\ Chris BARRY \\ University of Kent, Canterbury, England \\ and University of Essex, Colchester, England
}

\begin{abstract}
In the present study, we presented picture-naming latencies along with ratings for a set of important characteristics of pictures and picture names: age of acquisition, frequency, picture-name agreement, name agreement, visual complexity, familiarity, and word length. The validity of these data was established by calculating correlations with previous studies. Regression analyses show that our ratings account for a larger amount of variance in RTs than do previous data. RTs were predicted by all variables except complexity and length. A complete database presenting details about all of these variables is available in the supplemental materials, downloadable from http://brm.psychonomic-journals.org/content/supplemental.
\end{abstract}

Our identification of everyday objects is an ability that $j$ we all take for granted. Nonetheless, upon serious analysis, this ability turns out to be enormously complex. One way to learn about this process is to study how long it takes to name objects and to examine how different properties of pictures and their names affect this processing time. This kind of chronometric analysis of object identification has a long history in psychology. More recently, investigators have looked at the effects of a host of different variables on picture-naming times in order to understand how the process operates. Consider, for example, an influence of word frequency first reported by Oldfield and Wingfield (1965). Pictures whose names have a high frequency of occurrence in the language are named more quickly than those with low frequency names. This observation shows that the picture-naming system is sensitive to the frequency of the exposure of object names, and leads to the building of models of the lexicon in which lexical nodes have accumulated evidence for the presence of their referent object in the world. Higher frequency on this account is taken to influence this process by giving certain nodes a higher background or resting level of activation (McClelland \& Rumelhart, 1981), or by reducing the threshold for identification (Morton, 1969).

Researchers who are interested in the identification of natural objects face a number of difficult problems. For example, when exploring the identification of natural ob- jects, researchers cannot create stimuli that exactly match their requirements, but instead must exploit natural variation in the universe of existing objects. It is therefore important that researchers have access to information regarding the properties of a large set of stimuli. Snodgrass and Vanderwart (1980) were the first to systematically collect data on the properties of a set of pictures of objects. These norms and these pictures have been a valuable resource for cognitive psychologists who are interested in a range of issues that are concerned with how people process pictorial stimuli. Perhaps most importantly, these norms have permitted the development of factorial experiments using matched lists of stimuli. Experimenters were now able to manipulate variables of interest while holding other influential variables constant.

Despite the enormous contribution made by this pioneering study, there are a number of problems with the continued use of these stimuli. First, some of the variables that are known to influence picture naming are not static but change over time. Consider, for example, familiarity. The familiarity of everyday objects will almost certainly have changed between 1980 and the present day; some items that were very familiar back in 1980 may no longer be so, and vice versa (e.g., a record player vs. a cell phone). The same applies for other influential variables - for example, word frequency and age of acquisition (AoA).

R.A. Johnston, r.a.johnston@kent.ac.uk 
In addition to the problem of variables changing over time, there is another difficulty associated with conducting empirical investigations that is steadily increasing as researchers continue to identify a growing set of variables that are known to influence object identification. The burden on experimenters in designing factorial experiments is considerable because of the large number of variables that must be controlled. This problem is especially evident when one is trying to tease apart the influence of very closely interrelated variables. Consider the example of the variables AoA and frequency (see Johnston \& Barry, 2006, for a review). A recent study by Meschyan and Hernandez (2002) managed to disconfound these variables and generate item sets that were, for example, high in frequency but acquired later. However, they could not do this solely on the basis of the original Snodgrass and Vanderwart (1980) set of stimuli; it was necessary to use a considerably larger set to generate sufficient degrees of freedom. The task of researchers would be greatly eased if a larger set of pictures and norms existed in which all of those variables that were thought worth addressing were collected simultaneously.

Bates and colleagues (Bates et al., 2003; Bates et al., 2000; Székely et al., 2003; Székely et al., 2004) launched in the U.S. an ambitious project to establish norms for a large set of 520 pictures of objects for a range of languages. This project is most welcome and is reaching fascinating conclusions concerning cross-language consistency (see Bates et al., 2003). As well as driving novel theoretical conclusions, this research fulfills a more practical aim. Previously, there was little in the way of norms available outside of American English, although several studies have provided ratings for specific languages (Alario \& Ferrand, 1999, for French; Dell'Acqua, Lotto, \& Job, 2000, for Italian; Cuetos, Ellis, \& Alvarez, 1999, for Spanish). It is very important to establish sets of norms for different cultural populations because there will undoubtedly be variation in how particular items are viewed.

Bates and colleagues (Bates et al., 2003; Bates et al., 2000; Székely et al., 2003; Székely et al., 2004) have collected ratings for American English but not for British English. However, there are some important differences between American and British English. First, the names used to refer to the same object often differ between the two populations. If British researchers just take the U.S. norms, either they will have misleading expectations about the names of the objects in the minds of their participants, or they will lose many otherwise useful stimuli. Cultural differences also make us suspect that norms for other variables may depart between the two populations, especially familiarity and AoA. Given these concerns, it was decided to take the Bates et al. (2000) stimuli and to gather ratings on a range of dimensions from British English participants in order for researchers in the U.K. to have a good resource of a large set of pictures and norms.

One shortcoming of the set of stimuli assembled by Bates and her colleagues (Bates et al., 2003; Bates et al., 2000; Székely et al., 2003; Székely et al., 2004) is the relative preponderance of man-made artifacts at the ex-

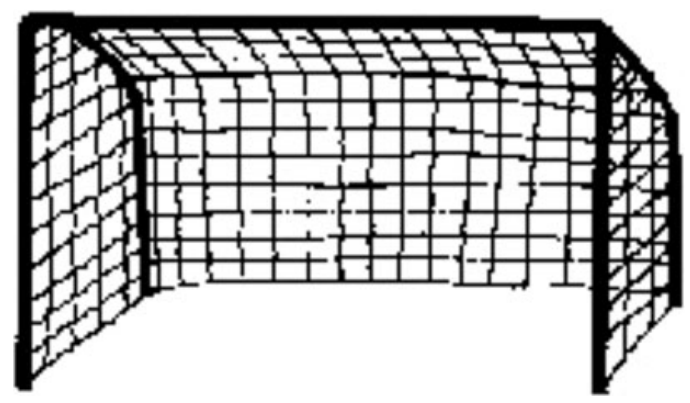

Figure 1. Example of the type of stimulus picture.

pense of living things, which probably reflects the actual composition of the real world. In order to partially address this imbalance in the original set, we augmented the stimuli with an additional 19 pictures of living things that were selected from a variety of sources. We hoped that this would make both the stimuli and norms of greater use to a wider audience, especially to those who are interested in category effects.

On the basis of previous research, we collected ratings for several variables that address important characteristics of pictures and their names. In addition, we collected naming latencies for the whole set of stimuli, using participants from the same population as the one that provided ratings. The resulting data allowed us to assess the relative importance of each of the variables in predicting naming latency. In addition, and in contrast with previous research, we assessed the possibility of interactions or interdependencies among the variables, focusing in particular on variables that index our experience with objects, frequency and AoA. These analyses revealed several important novel findings. In contrast with Ellis and Lambon Ralph's (2000) connectionist account of AoA effects, we found that AoA did not interact with a large number of other variables, indicating that AoA may be somewhat less pervasive than previously thought.

\section{PART 1}

\section{Collection of Ratings}

\section{Method}

Participants. A total of 31 staff and students of the University of Birmingham took part in this study for payment. Participants were paid $£ 5$ per hour for the time the task took to complete. All of the participants were paid either $£ 15$ or $£ 20$ in total. All were native English speakers who had lived continuously in the United Kingdom. They were all undertaking, or had already completed, a university degree. There were 21 females and 10 males whose average age was 21.6 years (the range was $19-32$ years).

Materials and Procedure. The set of drawings that was originally compiled by Bates et al. (2000) was used. All of the pictures were black-and-white line drawings that were like the item shown in Figure 1.

An additional 19 items that were taken from various sources were added to increase the number of living things in the set. Table 1 lists the original sources of the stimuli and the number of items taken from each source. All of the items, except those under the heading of miscellaneous, are available from the Web site for the International Picture Naming Project based at the University of Califor- 
nia, San Diego Center for Research in Language (http://crl.ucsd .edu/ aszekely/ipnp/). The remaining items are available on request from the first author.

A set of booklets was prepared that comprised the complete set of 539 pictures. Booklets consisted of 290 pages with a maximum of six objects presented per page. Three objects were presented on the left and three on the right; 289 pages contained six objects, and 1 page contained 5. Below each object appeared a list of the items of information to be provided by the participants. In the first slot, participants indicated the name of the object depicted. Participants then provided ratings for the following set of variables: AoA, picturename agreement (PNA), familiarity, and complexity. We will detail the specific instructions for rating each variable in the following paragraphs. However, the core procedure that was common to all variables was that all ratings were collected on a 7-point scale, the appropriate scale was presented, and participants circled a point on the scale accordingly.

A full set of instructions was presented to participants at the front of the booklet. When naming the items, participants were instructed to give the first name that came to mind, and to write DK ("don't know") if they did not know what the item was. They were instructed not to think too long about this and to avoid writing descriptions of the items.

Participants were also asked to rate the age at which they thought they had first learned the name of the object. They were instructed to rate the name and not the picture. The scale that was adopted followed that used by Gilhooly and Logie (1980). This comprised seven age bands, beginning with $0-2$ years old and increasing by 2 years at a time to 11-12 years old; the oldest response category was 13 years old or older. Participants were instructed to leave this rating blank if they had failed to name the item.

PNA rather than image agreement (IA) was collected. IA is usually measured by asking participants to generate a mental image in response to a name and then to rate the correspondence between their image and a subsequently displayed picture. However, our participants were instructed to ask themselves whether the picture provided a good example of the name, and to rate the extent to which the picture matched their expectations about how that concept should be depicted. This provided a measure of PNA. The scale ran from not at all as I would expect through to very much as I would expect.

Familiarity ratings followed the procedure used by Barry, Morrison, and Ellis (1997). Participants were asked to rate "how usual or unusual is the thing in your realm of experience" and to see familiarity as "the degree to which you come into contact with or think about the thing depicted." The scale ranged from very unfamiliar to highly familiar.

Participants also rated how complex they thought the drawing was. They were told that a complex drawing was one with many lines and angles, with a lot of detail and intricacy, and that a simple drawing was one with very few lines. The scale ran from very simple to very complex. A separate sheet provided examples of simple and complex drawings. The star from the Snodgrass and Vanderwart (1980) corpus was given as a simple drawing, and the "JCB" from the Bonin, Peereman, Malardier, Méot, and Chalard (2003) set was given as a complex drawing. Neither of the examples appeared for rating in the booklet. Two different orders of the pictures within the booklets were created, and the pages of each booklet were shuffled manually into a different order.

Participants were issued booklets that they took away and returned some time later. They were permitted to complete the booklet in their own homes, according to their own schedule, but they were encouraged to complete the task in a quiet environment. Participants reported that the task took between 2 and $4 \mathrm{~h}$ to complete, and they were paid accordingly, between $£ 15$ and $£ 20$.

\section{Compiling the Data}

From the data provided in the booklets, several variables were compiled. First, and most simply, we were able to de- termine the dominant name for our sample of participants. The dominant name is the name given most frequently by the participants. All spelling variations were treated as identical, but singulars and plurals were considered distinct names. A measure of name agreement was the proportion of responses that corresponded to the dominant name after those instances in which no response was provided had been excluded. AoA, familiarity, and PNA ratings were conditionalized on there being a correct, dominant response. There were two instances in which the dominant response was tied; in these cases, the experimenter exercised the casting vote. Complexity ratings were calculated from all available ratings, regardless of whether the dominant name was given, since the picture - not the name or conceptwas being rated. Lemma frequency for use as a noun was taken from the CELEX database (Baayen, Piepenbrock, \& Gulikers, 1995) for each item. A measure of word length was also included by counting the number of characters in each dominant name. An objective of particular interest had been the production of a data set with more living items than has been available in the past. The present set comprised pictures of 358 nonliving and 181 living items. The mean AoAs for the nonliving and living groups were $3.15(S D=0.84)$ and $3.14(S D=0.97)$, respectively. The mean familiarities for the nonliving and living groups were $4.94(S D=1.1)$ and $4.37(S D=1.2)$, respectively.

PART 2

\section{Timed Naming of the Picture Set}

\section{Method}

Participants. A total of 25 people took part in this experiment, and were paid $£ 5$ for their time. There were 21 females and 4 males. The mean age was 20.08 years (the range was $18-27$ years).

Materials and Equipment. A set of 544 pictures of objects was used in this study. Five items were pictures of geometric shapes and were used only as practice items. The remaining 539 pictures constituted the experimental items for which ratings had been obtained in the first part of this study. All items were black-and-white line drawings of everyday items presented against a plain white background. The maximum size of each picture was $300 \times 300$ pixels, but within these constraints, the size of each picture varied. The experiment was implemented using PsyScope experimental software (Cohen, MacWhinney, Flatt, \& Provost, 1993). Voice onset times were recorded using a Sony microphone connected to a PsyScope buttonbox, providing millisecond-accurate timing.

Procedure. Participants viewed and named all 544 pictures. Instructions were given verbally by the experimenter who was present throughout the session. Participants were instructed to name the item as soon as it appeared on the screen and to name each item as quickly as possible. They were informed that they would have a maximum time of $2 \mathrm{sec}$ in which to give their response. Participants first completed a block of practice trials employing a set of five geometric shapes. They then went on to complete four blocks of experimental trials with three blocks of 135 trials and one block of 134 trials. The experimenter initiated the sequence of trials. Each trial consisted of the following events: The screen was blank for $500 \mathrm{msec}$, and a fixation asterisk was then presented for $500 \mathrm{msec}$. The picture then appeared on the screen and stayed there until the participant made a response or $2 \mathrm{sec}$ had elapsed. The experimenter then initiated the next trial using the keyboard. Participants were permitted to rest at the end of each block.

Ten different orders of the items were created, and the participants were assigned to one of these orders. The experimenter recorded 
Table 1

Sources of Object-Naming Stimuli

\begin{tabular}{cc}
\hline Study Source & No. \\
\hline
\end{tabular}

Snodgrass \& Vanderwart (1980)

176

Alterations of Snodgrass \& Vanderwart (1980)

Peabody Picture Vocabulary Test (Dunn \& Dunn, 1981)

Alterations of Peabody Picture Vocabulary Test

Martinez-Dronkers set

Abbate \& La Chappelle (1984) "Pictures Please"

Max Planck Institute for Psycholinguistics

Boston Naming Test (Kaplan, Goodglass, \& Weintraub, 1983)

Oxford "One Thousand Pictures"

Morrison, Chappell, \& Ellis (1997)

Bonin, Peereman, Malardier, Méot, \& Chalard (2003)

Cycowicz, Friedman, Rothstein, \& Snodgrass (1997)

Miscellaneous

Note-All items except Miscellaneous are available from the International Picture Naming Project at the University of California, San Diego: http://crl.ucsd.edu/ aszekely/ipnp.

which name was spoken. The experimenter also recorded any problems with a trial, voice key failures, environmental triggerings, and verbal dysfluencies. The experimenter made a keypress to indicate whether any of the aforementioned events had invalidated a trial. The experimenter waited until the participant had completed his or her response before initiating the next trial.

\section{Compiling the Data}

Instances in which the voice key was triggered by an environmental sound, the voice key failed to trigger, a dysfluent response was given, or the participant took longer than $2 \mathrm{sec}$ to begin responding were excluded $(4.7 \%$ of the data). A response was included only if it corresponded to the dominant name as determined in the rating part of the study; this led to the exclusion of a further $18.3 \%$ of the data. We were also able to calculate a second measure of name agreement from the timed portion of the studythat is, the percentage of responses that corresponded to the dominant name as determined in the ratings portion of the study.

\section{ESTABLISHING VALIDITY}

\section{Intercorrelations With Previous Data Sets}

In order to gain an understanding of the relationships between the present data and previously published data sets, we calculated the correlations for each variable with four well-known published data sets. We explored the data sets presented by Barry et al. (1997), Székely et al. (2003), Snodgrass and Vanderwart (1980), and Morrison, Chappell, and Ellis (1997). Items were entered into these calculations only if the same picture had been used across the two studies and the same dominant name was given. The outcomes of these analyses can be seen in Table 2 .

\section{Discussion}

In the case of all variables, the present measures correlate significantly with the corresponding measures from each of the four previous studies in which corresponding data are available. This gives us confidence that the data reported in the present article are reliable and valid. Both AoA and familiarity are consistently very highly correlated across the studies. Percentage name agreement (\%NA) is overall less correlated than other variables. The present measure is somewhat different from that taken in Székely et al. (2003), since these authors calculated name agreement from data collected under timed naming conditions. In the present study, as in the other studies of interest, we calculated name agreement from unspeeded, self-paced written responses. Under unspeeded conditions in which participants are free to consider their responses at their leisure, they may be more likely to agree on the correct response and less likely to give an oddball response. However, such factors cannot account for the relatively low correlations between the present and past measures of name agreement from the other three studies, in which the same paper-and-pencil conditions were used. This may, to some extent, result from historical changes in the use of names, and it underscores the importance of up-to-date ratings data.

We calculated PNA rather than IA, largely for reasons of simplicity. PNA is a measure of the extent to which the picture is a good depiction of the object and meets with expectations about how it should be depicted. Our PNA measure correlates highly with the corresponding measure from Székely et al. (2003), who used a similar instruction. However, our measure of PNA correlates less well but still significantly with the data from the Snodgrass and Vanderwart (1980) and Morrison et al. (1997) norms. These last studies collected IA. The lower correlation between PNA and IA norms than between two measures of PNA shows that these two measures may be indexing somewhat different aspects of pictures and their names.

Our complexity data correlate very highly with those in other studies employing subjective ratings of complexity (Morrison et al., 1997; Snodgrass \& Vanderwart, 1980), but less highly with the one study employing objective complexity data, based on the file size in bytes (Székely et al., 2003; see Székely \& Bates, 2000, for further discussion of this measure.). This finding supports the argument made by Székely (Székely \& Bates, 2000; Székely et al., 2003) that objective and subjective ratings may differ in

Table 2

Comparisons With Previous Data Sets

\begin{tabular}{|c|c|c|c|c|c|c|}
\hline & AoA & $\mathrm{P}-\mathrm{N}$ & FAM & COMP & $\% \mathrm{NA}$ & RT \\
\hline \multicolumn{7}{|c|}{ Székely et al. (2003) } \\
\hline$r^{2}$ & $.876^{* * *}$ & $.694^{* * *}$ & $.906^{* * *}$ & $.582^{* * *}$ & $.520^{* * *}$ & $.819^{* * *}$ \\
\hline$n$ & 428 & 428 & 428 & 428 & 428 & 428 \\
\hline \multicolumn{7}{|c|}{ Barry, Morrison, \& Ellis (1997) } \\
\hline$r^{2}$ & $.931^{* * *}$ & & $.996^{* * *}$ & & $.737^{* * *}$ & $.742^{* * *}$ \\
\hline$n$ & 151 & & 166 & & 166 & 166 \\
\hline \multicolumn{7}{|c|}{ Snodgrass \& Vanderwart (1980) } \\
\hline$r^{2}$ & & $.373^{* * *}$ & $.878^{* * *}$ & $.923^{* * *}$ & $.622^{* * *}$ & \\
\hline$n$ & & 147 & 147 & 147 & 147 & \\
\hline \multicolumn{7}{|c|}{ Morrison, Chappell, \& Ellis (1997) } \\
\hline$r^{2}$ & $.944^{* * *}$ & $.516^{* * *}$ & $.928^{* * *}$ & $.903^{* * *}$ & $.702^{* * *}$ & \\
\hline$n$ & 142 & 142 & 142 & 142 & 142 & \\
\hline
\end{tabular}

Note-The data sets used for the comparisons were from Székely et al., 2003; Barry, Morrison, \& Ellis (1997); Snodgrass \& Vanderwart, 1980; and Morrison, Chappell, \& Ellis (1997). $n$, number of pictures. ${ }^{* * *} p<$ .001 . 
important ways, especially with respect to relationships with familiarity. Székely and Bates (2000) showed that, whereas subjective complexity ratings bear a nonsignificant relationship to familiarity, objective data do not.

Finally, our RT data correlate highly with those from both Székely et al. (2003) and Barry et al. (1997) (even though participants in the Barry et al. study named about half as many objects in a single session). In agreement with Székely et al. (2003), this shows that participants can name several hundred objects in a single session without substantial loss in data quality. The previous analysis establishes the validity of the present data; researchers can have confidence in using these data.

\section{INTERCORRELATIONS AMONG THE VARIABLES}

Correlations between the following set of variables were calculated: AoA; PNA; familiarity; complexity; number of alternative names; $\mathrm{H}$ statistic (a measure of response agreement that considers the proportion of subjects producing each alternative: Higher values of $\mathrm{H}$ indicate lower values of name agreement); \%NA untimed; \%NA timed; number of characters; spoken, written, and combined CELEX lemma frequency; raw RT; and harmonic means of RT. This analysis was also conducted in order to identify a core set of predictor variables for entry into multiple regression. In multiple regression, it is desirable to enter a set of variables that are as nonredundant as possible, in order to avoid problems of multicollinearity. At this stage, several highly related variables were included: several measures of frequency and several measures of "codability" (i.e., several measures of the extent to which an item is given the same name by the participants). For the regression analysis, only one variable from each group was taken forward, the criteria for selection being the variable that correlated most highly with RT but with the smallest number of other variables. The complete pattern of correlations may be seen in the Appendix.

AoA has been identified as a key predictor of lexical processing speed (see, e.g., Brown \& Watson, 1987; Gerhand \& Barry, 1998; Gilhooly \& Logie, 1981; Morrison \& Ellis, 1995; Rubin, 1980); hence, we paid particular attention to this variable. AoA correlated significantly with all other variables, apart from three of the measures of "codability" $(\mathrm{H}$, number of alternatives, \%NA untimed); AoA did correlate significantly with \%NA timed. This correlation shows that late-acquired items are less well named under speeded conditions; however, under unspeeded conditions, late-acquired items are not any less likely than early ones to be given the same name across participants. It is likely that under speeded conditions, participants sometimes terminate processing prematurely and, when this occurs, because the late items require longer to process, they are more vulnerable to error.

The four measures of codability correlated highly with one another. Name agreement calculated from timed data correlated significantly with all other variables. The other three measures of name agreement that were calculated from the untimed portion of the study correlated with far fewer variables, and correlated significantly only with PNA and RT. This pattern of correlations suggests that timed name agreement is largely a redundant variable, and that it is not such a good and pure measure of codability. The other measures seem to pick up something unique about lexical processing that is not accounted for by other variables, but all of these three measures of codability are largely interchangeable. For further analysis, we included only \%NA untimed. Under speeded conditions, a range of variables affects the number of errors; however, under unspeeded conditions, these same variables do not predict name disagreement. Across studies, different researchers have employed different measures of name agreement, sometimes choosing to measure speeded or unspeeded versions. These data illustrate that there are important contrasts between these two different versions of what is ostensibly the same variable.

All three measures of frequency are highly intercorrelated and show the same pattern of relationships across the other variables. It would appear that spoken and written frequency counts are largely interchangeable. Similar to AoA, frequency is unrelated to the pure measures of codability identified previously; however, unlike AoA, it is also unrelated to to PNA.

Much has been made in the literature of the relationships between AoA and word frequency: The two variables might reflect the same factor, or they may influence distinct stages of lexical processing. Although the present pattern of intercorrelations displays a very similar pattern for the two variables, there are important differences. Whereas AoA is significantly related to PNA, frequency is not. Later acquired concepts tend to be rated as showing poorer PNA, but there is no difference in PNA for low-frequency and high-frequency items. PNA itself is related to everything apart from all measures of word frequency and familiarity. Complexity is related to everything else apart from pure measures of codability. Familiarity is related to everything else apart from PNA and measures of codability.

\section{FACTOR ANALYSIS}

A factor analysis was performed in order to further explore the structure in the set of predictor variables. The outcome of this analysis may be seen in Table 3 . Two factors accounted for $56.35 \%$ of the variance. This analysis

Table 3

Factor Analysis of Data Set

\begin{tabular}{|c|c|c|}
\hline Variable & Factor 1 & Factor 2 \\
\hline Age of acquisition & 0.840258 & -0.015133 \\
\hline Picture-name agreement & -0.195520 & -0.784631 \\
\hline Familiarity & -0.796090 & 0.034718 \\
\hline Complexity & 0.506536 & 0.363017 \\
\hline$\%$ Name agreement & -0.093549 & -0.644138 \\
\hline Length & 0.586813 & -0.246769 \\
\hline Frequency & -0.815026 & 0.260592 \\
\hline Variance & 0.378853 & 0.184654 \\
\hline
\end{tabular}


supports the existence of two major groups of predictors in this set. The first group is represented by Factor 1, which has very high loadings from AoA, familiarity, and frequency; the second group has high loadings from PNA and name agreement. The first factor picks up a highly intercorrelated set of variables that all reflect aspects of experience with objects and their names. The three variables - familiarity, frequency, and AoA - are more highly correlated with each other than they are with the remaining predictor variables. The second factor shows that name agreement and PNA are more closely related to each other than they are to the rest of the variables, probably because PNA is a strong determinant of naming success. When an object is depicted in a way we expect, we are more likely to agree on its name. Furthermore, this implies that instances of name disagreement arise not only because an object has more than one legitimate name, but also because the object may be misidentified as something it is not.

\section{COMPARISONS WITH PREVIOUS STUDIES}

In the next section we compare our pattern of correlations with those obtained in two other studies: Székely et al. (2003) and Barry et al. (1997).

\section{Székely et al. (2003)}

The most salient difference arises with respect to complexity. Székely et al. (2003) measured objective complexity by taking the file size of each image in bytes as their measure. This measure of complexity was related only to length. Their measure of name agreement calculated from timed data was related to many more variables and was not significantly related only to length and complexity. The Székely et al. measure of PNA was significantly related to a range of variables and behaved similarly to the current measure of PNA, being related to everything apart from frequency, length, and complexity. Also, in these data, AoA was significantly related to measures of codability, number of alternatives, and name agreement. Our measure of AoA was not. This difference likely reflects the fact that Székely et al.'s measures correspond to speeded conditions, whereas our \%NA corresponds to unspeeded conditions.

\section{Barry et al. (1997)}

We considered the pattern of correlations for the set of 195 items included in the Barry et al. analysis. A number of salient differences emerged. The measure of image agreement included by Barry et al. (taken from Snodgrass \& Vanderwart, 1980) showed no significant correlation with

Table 4

Relationship to Previous Intercorrelations

\begin{tabular}{lc}
\hline \multicolumn{1}{c}{ Data } & $R^{2}$ \\
\hline Barry, Morrison, \& Ellis (1997) $\rightarrow$ Barry et al. (1997) & .4589 \\
Barry et al. (1997) $\rightarrow$ Present data & .4043 \\
Present data $\rightarrow$ Barry et al. (1997) & .6266 \\
Present data $\rightarrow$ Present data & .5387 \\
\hline
\end{tabular}

Table 5

Regression Analysis on Naming Reaction Time

\begin{tabular}{lrcrc}
\hline \multicolumn{1}{c}{ Variable } & Beta & $S E$ of Beta & \multicolumn{1}{c}{$t(393)$} & $p$ \\
\hline Age of acquisition & .341 & .048 & 7.106 & $<.001$ \\
Picture-name agreement & -.447 & .035 & -12.609 & $<.001$ \\
Familiarity & -.117 & .048 & -2.452 & .015 \\
Complexity & -.010 & .037 & -0.278 & .780 \\
\% name agreement & -.148 & .034 & -4.291 & .0000224 \\
Length & .033 & .040 & 0.827 & .409 \\
Frequency & -.101 & .049 & -2.040 & .042 \\
\hline
\end{tabular}

any of the other variables. In contrast, our measure of PNA was significantly correlated to several other variables. Furthermore, the Barry et al. AoA ratings were significantly related to their name agreement scores; however, our AoA ratings were not. Finally, although Barry et al.'s nameagreement scores were significantly related to measures of word length, ours were not.

We also considered the relationships between the Barry et al. (1997) data and the present data in a series of four regression analyses, in which we considered the 151 items in common between the two sets, in which the same name was given and frequency data were available (see Table 4). In the first two analyses, we took Barry et al.'s RTs and predicted them from both their own (Analysis 1) and our (Analysis 2) rating data. In the second two analyses, we took our RTs and predicted them from both Barry et al.'s (Analysis 3) and our (Analysis 4) rating data.

From the outcome of the set of analyses shown in Table 4, it can be seen that although RTs from Barry et al. (1997) are better predicted than our RTs from both sets of ratings, our norms better predict both sets of RTs. In all of these analyses, harmonic means rather than simple arithmetic means were used, since only harmonic mean data were available from the Barry et al. study.

The previous set of analyses show that our rating data are more powerful in predicting RT than are the Barry et al. (1997) ratings.

\section{REGRESSION ANALYSES}

On the basis of the above correlational analyses, we chose to include the following variables in a regression analysis with picture-naming time as the dependent variable: AoA, ln (natural logarithm) frequency CELEX combined, PNA, familiarity, complexity, name agreement, and word length (number of letters).

\section{Results and Discussion}

Analyses predicting mean RT and harmonic RT were compared, and the predictor variables accounted for a larger amount of variance in mean RT than in harmonic mean RT. We therefore present the data for mean RT below. The outcomes of this regression analysis may be seen in Table 5. All of the variables were forced into a simultaneous multiple regression analysis. Overall, the regression equation captured a significant proportion of the variation in RTs $\left(R^{2}=.565\right)$. Only two variables were not significant predictors of naming RT: visual complexity and word 
length. In examining those variables that were significant predictors, we found that PNA was the most important, closely followed by AoA. Name agreement, familiarity, and frequency were all similar in terms of importance and were considerably weaker than AoA and PNA. This pattern of findings is similar to that generated by Barry et al. (1997); however, there are some important differences. In addition to visual complexity and word length, in the Barry et al. analysis, image agreement and familiarity were nonsignificant. In the present analysis, both PNA and familiarity were significant predictors, presumably reflecting the larger set of items included in our set.

\section{GENERAL DISCUSSION}

The previous analyses establish the validity of the norms presented here. The present data correlate highly with the norms presented in other well-known studies, and they provide a better account of picture-naming times (see Barry et al., 1997). It can be seen from Table 2 that our ratings correlate more highly with data collected in the two British studies than with data collected in the U.S. These norms can thus serve as a resource for researchers in the U.K. working on object naming with British-English participants. The present data have further value, since we presented statistics for more than twice as many items as in the previous U.K. studies.

The corpus of data collected allowed us to address the nature of both AoA and frequency effects by considering possible interactions with other variables. Popular interpretations of the Ellis and Lambon Ralph (2000) neural network implementation of AoA effects suggest that AoA effects should be found throughout the object identification system. One might therefore expect to find interactions between AoA and a range of other important variables, but this was not the case in the present data set. Variables that have a clear-cut and independent effect on naming time were not shown to interact with AoA. In particular, PNA and name agreement both failed to interact with AoA. Although it is important to note that these observations reflect null effects, such findings have important consequences for understanding AoA. Future research should attempt to systematically manipulate AoA in combination with the orthogonal manipulation of other important variables to examine their combined effect on picture-naming latencies. It might be the case that AoA effects are less widespread than has been previously thought.

The data collected in the present study are available for download from the Psychonomic Society supplemental materials archive. An Excel file contains ratings for all 539 stimuli employed in the study. It is indexed in relation to the British-English names for stimuli, but we also include a listing of the U.S. English alternatives. One worksheet contains all of the ratings relating to our various measures of AoA, familiarity, PNA, complexity, frequency, and codability (i.e., name agreement). A second worksheet contains the mean correct picture-naming RTs for all stimuli, along with the individual responses for all 25 participants. It also contains a breakdown of the failed responses as voicekey failures, environmental triggerings, or verbal dysfluencies.

\section{AUTHOR NOTE}

Correspondence concerning this article should be addressed to R. A Johnston, School of Psychology, Keynes College, University of Kent, Canterbury CT2 7NP, England (e-mail: r.a.johnston@kent.ac.uk).

\section{REFERENCES}

Abbate, M. S., \& La Chappelle, N. B. (1984). Pictures, please! A language supplement. Tucson: Communication Skill Builders.

Alario, F.-X., \& Ferrand, L. (1999). A set of 400 pictures standardized for French: Norms for name agreement, image agreement, familiarity, visual complexity, image variability, and age of acquisition. Behavior Research Methods, Instruments, \& Computers, 31, 531-552.

BaAyen, R. H., Piepenbrock, R., \& Gulikers, L. (1995). The CELEX lexical database [CD-ROM]. Philadelphia: University of Pennsylvania, Linguistic Data Consortium.

Barry, C., Morrison, C. M., \& Ellis, A. W. (1997). Naming the Snodgrass and Vanderwart pictures: Effects of age of acquisition, frequency and name agreement. Quarterly Journal of Experimental Psychology, 50A, 560-585.

Bates, E., D'Amico, S., Jacobsen, T., SzéKely, A., Andonova, E., DeVESCOVI, A., ET AL. (2003). Timed picture naming in seven languages. Psychonomic Bulletin \& Review, 10, 344-380.

Bates, E., Federmeier, K., Herron, D., Iyer, G., Jacobsen, T., PechMANN, T., ET AL. (2000). Introducing the CRL International PictureNaming Project (CRL-IPNP). Center for Research in Language Newsletter, 12. La Jolla: University of California.

Bonin, P., Peereman, R., Malardier, N., Méot, A., \& Chalard, M. (2003). A new set of 299 pictures for psycholinguistic studies: French norms for name agreement, image agreement, conceptual familiarity, visual complexity, image variability, age of acquisition, and naming latencies. Behavior Research Methods, Instruments, \& Computers, 35, 158-167.

Brown, G. D., \& Watson, F. L. (1987). First in, first out: Word learning age and spoken word frequency as predictors of word familiarity and word naming latency. Memory \& Cognition, 15, 208-216.

Cohen, Jonathan [D.], MacWhinney, B., Flatt, M., \& Provost, J. (1993). PsyScope: An interactive graphic system for designing and controlling experiments in the psychology laboratory using Macintosh computers. Behavior Research Methods, Instruments, \& Computers, 25, 257-271.

Cuetos, F., Ellis, A. W., \& Alvarez, B. (1999). Naming times for the Snodgrass and Vanderwart pictures in Spanish. Behavior Research Methods, Instruments, \& Computers, 31, 650-658.

Cycowicz, Y. M., Friedman, D., Rothstein, M., \& Snodgrass, J. G. (1997). Picture naming by young children: Norms for name agreement, familiarity and visual complexity. Journal of Experimental Child Psychology, 65, 171-237.

Dell'AcQua, R., Lotto, L., \& Joв, R. (2000). Naming times and standardized norms for the Italian PD/DPSS set of 266 pictures: Direct comparisons with American, English, French, and Spanish published databases. Behavior Research Methods, Instruments, \& Computers, 32, 588-615.

Dunn, L. M., \& Dunn, L. M. (1981). Peabody Picture Vocabulary Test—Revised. Circle Pines, MN: American Guidance Service.

Ellis, A. W., \& Lambon Ralph, M. A. (2000). Age of acquisition effects in adult lexical processing reflects loss of plasticity in maturing systems: Insights from connectionist networks. Journal of Experimental Psychology: Learning, Memory, \& Cognition, 26, 1103-1123.

GERHAND, S., \& BARRY, C. (1998). Word frequency effects in oral reading are not merely age-of-acquisition effects in disguise. Journal of Experimental Psychology: Learning, Memory, \& Cognition, 24, 267-283.

Gilhooly, K. J., \& Logie, R. H. (1980). Age-of-acquisition, imagery, concreteness, familiarity and ambiguity measures for 1,944 words. Behavior Research Methods \& Instrumentation, 12, 395-427.

Gilhooly, K. J., \& Logie, R. H. (1981). Word age-of-acquisition, reading latencies and auditory recognition. Current Psychological Research, 1, 251-262. 
Johnston, R. A., \& BARry, C. (2006). Age of acquisition and lexical processing: A review. Visual Cognition, 13, 789 -845.

Kaplan, E., Goodglass, H., \& Weintraub, S. (1983). Boston Naming Test. Philadelphia: Lee \& Febiger.

McClelland, J. L., \& Rumelhart, D. E. (1981). An interactive activation model of context effects in letter perception: I. An account of basic findings. Psychological Review, 88, 375-407.

Meschyan, G., \& Hernandez, H. (2002). Age of acquisition and word frequency: Determinants of object-naming speed and accuracy. Memory \& Cognition, 30, 262-269.

Morrison, C. M., Chappell, T. D., \& Ellis, A. W. (1997). Age of acquisition norms for a large set of object names and their relation to adult estimates and other variables. Quarterly Journal of Experimental Psychology, 50A, 528-539.

Morrison, C. M., \& Ellis, A. W. (1995). Roles of word-frequency and age of acquisition in word naming and lexical decision. Journal of Experimental Psychology: Learning, Memory, \& Cognition, 21, 116-133.

Morton, J. (1969). Interaction of information in word recognition. Psychological Review, 76, 165-178.

OldFiELD, R. C., \& WingField, A. (1965). Response latencies in naming objects. Quarterly Journal of Experimental Psychology, 4, 272-281.

RuBIN, D. C. (1980). 51 properties of 125 words: A unit analysis of verbal behavior. Journal of Verbal Learning \& Verbal Behavior, 19, 736-755.

SNODGRass, J. G., \& VANDERWART, M. (1980). A standardized set of 260 pictures: Norms for name agreement, image agreement, familiarity, and visual complexity. Journal of Experimental Psychology: Human Learning \& Memory, 6, 174-215.

Snodgrass, J. G., \& Yuditsky, T. (1996). Naming times for the Snodgrass and Vanderwart pictures. Behavior Research Methods, Instruments, \& Computers, 28, 516-536.

SzÉKeLY, A., \& BATES, E. (2000). Objective visual complexity as a variable in studies of picture naming. Center for Research in Language Newsletter, 12(2). La Jolla: University of California.

Székely, A., D’Amico, S., Devescovi, A., Federmeier, K., HerRON, D., IYER, G., ET AL. (2003). Timed picture naming: Extended norms and validation against previous studies. Behavior Research Methods, Instruments, \& Computers, 35, 621-633.

Székely, A., Jacobsen, T., D'Amico, S., Devescovi, A., Andonova, E., HERRON, D., ET AL. (2004). A new online resource for psycholinguistic studies. Journal of Memory \& Language, 51, 247-250.

\section{SUPPLEMENTAL MATERIALS}

The full norms and naming times for the 539 pictures discussed in this article may be downloaded from http://brm.psychonomic-journals .org/content/supplemental.

(Manuscript received July 5, 2009;

revision accepted for publication December 6, 2009.) 


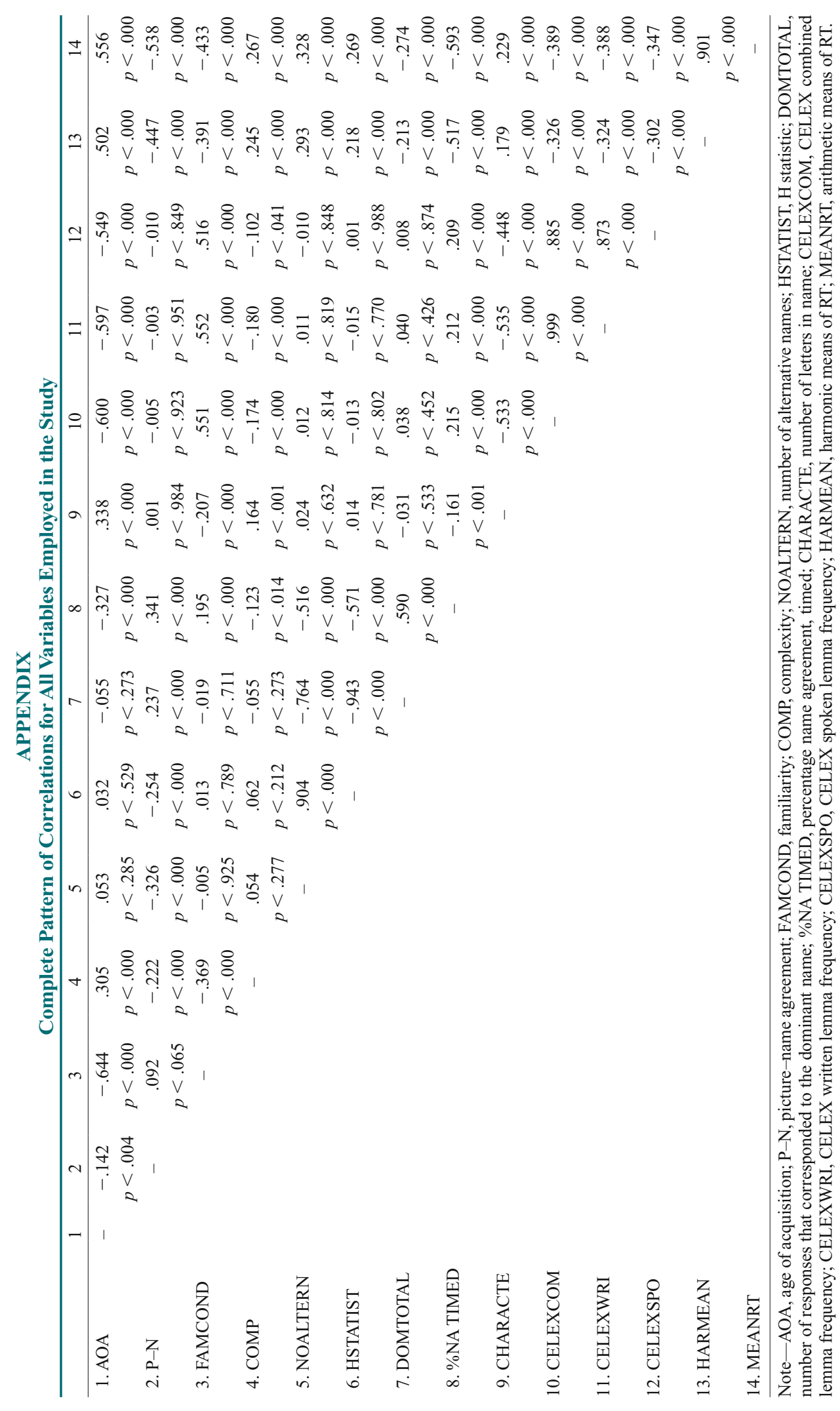

